\title{
Estrategias de la gestión de calidad en las empresas mixtas venezolanas
}

\section{Strategies of quality management in venezuelan mixed companies}

\author{
Ygcibel Garrido \\ ygcibel.cecilia@gmail.com \\ ORCID: 0000-0003-3729-2660 \\ Universidad del Zulia, Zulia - Venezuela \\ Artículo recibido en febrero 2020 / Arbitrado en marzo 2020 / Aceptado en junio 2020 / Publicado en julio 2020
}

Se identificaron las estrategias de la gestión de calidad que aplican las empresas mixtas venezolanas. La metodología fue descriptiva, con diseño no experimental, de campo y transeccional. La población quedó constituida por las gerencias de yacimientos pertenecientes a las tres empresas mixtas filiales de PDVSA que hacen vida activa en la Costa Oriental del Lago. Para la recolección de datos se empleó la encuesta mediante un cuestionario, conformado por 10 ítems con escala de frecuencia. La validez se realizó a través del juicio de expertos, y en su confiabilidad se empleó el Coeficiente Alfa de Cronbach, obteniéndose 0,812. El análisis de los datos se realizó con estadística descriptiva, basado en la media aritmética. Se concluye que: estas gerencias ostentaron baja aplicación de la planificación de la calidad, diseño de la calidad, despliegue funcional, análisis modo de fallas y efectos, control de los procesos, como estrategias de la gestión de calidad.

Palabras clave: Análisis modo de fallas y efectos; control de los procesos; despliegue funcional, diseño de la calidad; estrategias de la gestión de calidad; planificación de la calidad

The quality management strategies applied by Venezuelan joint ventures were identified. The methodology was descriptive, with a non-experimental, field and transectional design. The population was made up of reservoir managers belonging to the three PDVSA affiliated joint ventures that make active life on the Eastern Coast of the Lake. For data collection, the survey was used using a questionnaire, made up of 10 items with a frequency scale. Validity was carried out through expert judgment, and Cronbach's Alpha Coefficient was used in its reliability, obtaining 0.812. Data analysis was performed with descriptive statistics, based on the arithmetic mean. It is concluded that: these managements had low application of quality planning, quality design, functional deployment, failure and effect mode analysis, process control, as quality management strategies.

Keywords: Failure mode and effects analysis; process control; functional deployment; quality design; quality management strategies; quality planning 


\section{INTRODUCCIÓN}

La gestión de la calidad hoy en día traspasa las fronteras del entorno empresarial y arropa todo el conjunto de instituciones establecidas en la sociedad, sobre todo aquellas dedicadas a las actividades del sector petrolero. En este sentido, estas organizaciones enfrentan el reto de ofrecer excelencia y calidad en su gestión, como elementos de competitividad que les permitan garantizar su permanencia y evolución en el tiempo, satisfaciendo las necesidades y expectativas de sus clientes.

Bajo esta perspectiva, para Gutiérrez (2010), la implementación de un sistema de gestión de calidad, debe estar basado en estrategias que establecen unos principios a seguir, que indican qué debe hacerse pero sin explicar cómo debe hacerse, puesto que esto último se describe en un procedimiento.

En este contexto, a criterio de Collado (2006), mundialmente, los países productores de petróleo cada día se hacen más competitivos dentro de sus funciones de generar energía; las organizaciones que materializan este mercado se encuentran en constantes cambios, ya que el crecimiento de la humanidad y la demanda de energía las obligan a aplicar nuevas estrategias que involucran procesos centrados en lograr la calidad en sus operaciones y en lo que esta involucra.

De manera específica, según Marín (2009), uno de los sectores que ha puesto mayor énfasis en la mejora continua de sus procesos está representado por las empresas de hidrocarburos liderizadas por Petróleos de Venezuela S.A. (PDVSA) y empresas filiales, las cuales se han visto obligadas a analizar las debilidades adquiridas por sus factores productivos, culturales y tecnológicos, con la finalidad de evaluar la competitividad de su estructura; cómo están definidos sus índices de gestión a favor de la productividad; si se están cumpliendo las normas de calidad; si existen programas de mantenimiento, garantizando la continuidad operacional de sus procesos, entre otros aspectos.

Bajo este enfoque, según información dada de manera informal por los líderes de calidad de las gerencias de yacimientos en las empresas mixtas venezolanas de la Costa Oriental del Lago, se pudo evidenciar como estas gerencias carecen de un sistema para la elaboración, difusión y revisión de las normas, técnicas, procedimientos del sistema de calidad, igualmente no tienen definidos los criterios para decidir la aplicación de las auditorias de calidad requeridas, al igual que no se tiene establecido un programa de auditorías internas o externas, que considere las diferentes actividades a seguir para el desarrollo de las normas, no se informa las deficiencias o necesidades.

De manera similar, de acuerdo a la percepción de la investigadora, por ser parte activa de las mismas, se evidenciaron fallas en cuanto a la calidad de los procesos, dado que en ocasiones el personal no posee las competencias necesarias para ejecutar sus funciones sobre la base de los lineamientos de calidad exigidos en la normativa legal vigente, repercutiendo en la continuidad operacional.

Esta situación, a juicio de la investigadora, pudiese ser producto de que estas empresas poseen estructuras organizativas de una relativa reciente creación, lo cual les ha dificultado el establecimiento de un sistema de gestión de calidad acorde a la envergadura de sus operaciones. De igual manera, no se evidenciaron estrategias para emplear la gestión de calidad, dado que no emplean mecanismos formales para lograr el funcionamiento óptimo de las maquinarias y equipos, situación que en ocasiones genera retrasos de los procesos. 
Por tal razón, surgió la inquietud de identificar las estrategias de la gestión de calidad que aplican las empresas mixtas venezolanas y que hacen vida activa en la Costa Oriental del Lago, a fin de contribuir a la organización de sus procesos y mejorar el desempeño, constituyéndose en una herramienta de acción para el logro de sus objetivos, donde la calidad en sus productos y servicios permita a estas gerencias cumplir con las metas establecidas y satisfacer los requerimientos del entorno.

Según Gutiérrez (2010) se pueden definir las estrategias de calidad como una guía para la actividad de la dirección donde se plasman los principios de la calidad para la empresa, detallando entre otras, la intención de satisfacer las necesidades del cliente, las necesidades concretas que han de satisfacerse y la mejora de la calidad. Afirma este autor que, las estrategias de calidad pueden ser establecidas en cada organización de acuerdo a los objetivos trazados en cuanto a calidad se refiere, siendo las más usuales: planificación de la calidad, diseño de la calidad, despliegue funcional, análisis modo de fallas y efectos y el control de los procesos.

Considerando estas definiciones, Arthur (2010) postula que, es mejor tener una política en lugar de un procedimiento con el fin de proporcionar a la empresa la flexibilidad necesaria para adaptarse rápidamente a situaciones diferentes. Sin embargo, es habitual que en la empresa existan ambos, de forma que los procedimientos se redactan teniendo en cuenta que no deben suponer mayor rigidez para la organización.

En esta perspectiva, para Benavides (2008), los objetivos deben ser incorporados al plan del negocio, y por tanto la alta administración debe involucrarse personalmente en esta planificación y determinar los objetivos que se incluirán, para posteriormente desplegarlos a los niveles inferiores. Por consiguiente, la alta administración debe identificar las acciones a desarrollar para alcanzar los objetivos, proporcionar recursos para ello y asignar quién es el responsable de las mismas.

Dentro de estos objetivos estratégicos, afirma el autor citado, la empresa puede desear conseguir un premio a la calidad. En este sentido, a nivel nacional e internacional existe un reconocimiento institucional al notable esfuerzo que algunas empresas realizan en la implantación de programas de mejora de la calidad a través de una serie de premios. En estos casos, las organizaciones ganadoras de un premio de calidad pueden comunicar el mismo a sus clientes como prueba de que han mejorado la misma (Hendricks y Singhal, 2006).

En este sentido, la evidencia empírica demuestra cómo el valor de las acciones de empresas ganadoras de premios de este tipo reacciona positivamente a los anuncios que hacen éstas sobre el premio de calidad conseguido, reacción particularmente fuerte en el caso de empresas pequeñas.

Ahora bien, de acuerdo a lo expuesto, la investigadora asume que las estrategias de calidad deben indicar el camino a seguir para que la dirección de la organización pueda encaminar los esfuerzos al logro de los objetivos de calidad, son una guía para la actividad de la dirección en busca de la calidad, con la intención de satisfacer las necesidades del cliente.

A manera de abordar el estudio de este punto, la investigadora coincide con el postulado de Gutiérrez (2010), en cuanto a las estrategias de la gestión de calidad más usuales, y que deben aplicar las gerencias de yacimientos de las empresas mixtas bajo estudio. Por ello, los indicadores para medir 
esta dimensión serán los mencionados por este autor: planificación de la calidad, diseño de la calidad, despliegue funcional, análisis modo de fallas y efectos y el control de los procesos.

\section{Planificación de la calidad}

Analizar los casos de la realidad de manera objetiva, colocar metas posibles para que sea realista, según el criterio de Arthur (2010), e s una etapa clave en el proceso gerencial denominada planificación, aquí se decide a donde debe ir la organización y como puede llegar allí, mediante la evaluación del entorno, apoyando el proyecto de acción con el conocimiento pleno de las fortalezas y debilidades de la organización. De esta definición, se deduce que se planifica para atender necesidades individuales y sociales que determinan el comportamiento eficaz y de calidad, tanto del personal administrativo como de los clientes.

Benavides (2008), dice, planear implica tomar las decisiones más adecuadas acerca de lo que habrá de realizarse en un futuro, establece las bases para determinar el elemento riesgo y minimizarlo. La eficiencia en la ejecución depende en gran parte de una adecuada planeación, y los buenos resultados no se logran por sí mismos, se requiere establecerlos con anticipación. Al respecto, la planeación es la herramienta que ayuda a los administradores a desarrollar nuevas aptitudes y procedimientos para encarar los negocios de mañana. Aunque también proporciona a la empresa muchas otras cosas valiosas, su mayor aportación radica en hacer frente al cambio: de los negocios de hoy a los mañana.

Por otro lado, James (2010), expone que la planificación es la primera función de la dirección, se orienta al futuro y crea las directrices para toda la organización, por lo tanto, su importancia no debe dejar de ser acentuada, ya que si no se planifica en una organización se pierde recursos, energías y reputación. En este sentido, la planificación ofrece la capacidad de ser proactivos $y$ anticipar futuros eventos, estableciendo las acciones necesarias para enfrentar positivamente a ellos.

En el terreno de la calidad, para Cuatrecasas (2010) la correcta gestión de la misma supone la planificación, diseño, desarrollo de productos y procesos en el marco de una organización, así como la gestión de los recursos humanos para la calidad, además, de la adecuada implantación y control de calidad para su certificación final. Todo ello supondrá una gestión de la empresa, donde sus productos y procesos, basados en la calidad, llevarán a la misma a obtener el máximo de ventajas competitivas, además, de la satisfacción total de los clientes mediante la identificación, aceptación y satisfacción de todas sus expectativas.

Según la Norma ISO 9001: 2008, en la planificación de la calidad se establecen los objetivos y procesos de acuerdo con los requerimientos y políticas de la organización. Para Muñoz (2009), la planificación de la calidad está enfocada a diseñar productos y procesos que satisfagan las necesidades de los clientes. Mientras, a juicio de Koontz y Weihrich (2009), planificar implica la selección de misiones, objetivos y acciones para llevar a cabo las primeras y alcanzar los segundos, requiere tomar decisiones, esto es, elegir entre alternativas de futuros cursos de acción.

Al respecto, Pérez y Munera (2007), explican que, el planificar será mucho más fácil si se definen los objetivos de lo que se quiere alcanzar, y es más fácil también pues se puede verificar si lo que se hizo corresponde a lo planeado. Cabe destacar que, una mala 
planificación no permite una buena ejecución y tampoco una correcta verificación. Por tanto, la etapa de planificación es clave. Para muchos autores la planificación incluye mucho de capacitación implica el entrenamiento previo a la ejecución.

De acuerdo con los autores citados, la investigadora expresa que, se debe realizar una efectiva planificación donde se conozcan los objetivos de una manera clara, donde se puede hacer un control preciso de cada paso dentro del proceso de la gestión de la calidad de la organización. En la fase de planificación se buscan las actividades susceptibles de mejora y se establecen los objetivos a alcanzar. Para buscar posibles mejoras se pueden realizar grupos de trabajo, buscar asesorías de proveedores, escuchar las sugerencias de los clientes y las opiniones de los trabajadores, buscar nuevas tecnologías mejores a las que se están usando ahora, entre otras.

\section{Diseño de la calidad}

Para Gutiérrez (2010) en un ambiente de calidad, la identificación y resolución de problemas debe ser la práctica habitual en el traba jo diario que puede estar escrito en la descripción del puesto de trabajo. No obstante, en realidad, las empresas han realizado desde siempre actividades que identificaban los problemas como parte natural de su funcionamiento.

En el caso de las organizaciones de calidad, afirma el autor citado, la identificación de los problemas se realizaba mediante las actividades tradicionales de control como la planificación y auditorías de control del desempeño, las relaciones con los clientes, los programas de involucramiento de los empleados. Por tanto, la identificación de problemas es una parte de la dirección de calidad y de la práctica de la administración en general, siendo una actividad que debe ser administrada como la planificación, la evaluación del desempeño y otras actividades organizativas.

Según Arthur (2010), los empleados pueden intervenir en las actividades de identificar problemas, si tienen los conocimientos y las técnicas adecuadas. Así, se requiere una formación específica, la cual debe estar centrada, por un lado, en temas generales de calidad y, por otro, debe abarcar las principales herramientas de trabajo en equipo y la metodología de grupo para la resolución de problemas. Existen para ello técnicas sencillas y avanzadas, fundamentadas en unas herramientas específicas que facilitan el trabajo de los participantes, con la finalidad de desarrollar actividades de análisis, diagnóstico, definición de líneas de actuación, implantación $\mathrm{y}$ ajuste.

Entre las diversas técnicas de mejora de la calidad, las herramientas estadísticas básicas son las más sencillas y utilizadas por los operarios. Con estas actividades los individuos y la organización pueden aprender, lo que lleva posteriormente a mejorar. De esta manera, la mejora de la calidad va a depender, en gran medida, de las personas implicadas en dichas actividades. Se puede afirmar que la dirección de la calidad supera en gran medida a la certificación de los sistemas de aseguramiento externo (ISO 9000) que sigue siendo un objetivo de gran prioridad en muchos sectores.

Sin embargo, cada vez más las empresas, organizaciones de apoyo, organizaciones profesionales, consultores y universidades, comprenden la importancia esencial pero relativa de la certificación de los sistemas de calidad. Como consecuencia, la certificación se contempla como el punto de partida en el camino hacia la calidad total (Van Ham, 2009). 
De esta manera, aunque una empresa puede implantar un sistema de dirección de la calidad sin obtener la certificación, en la práctica la obtención de uno de estos certificados sí puede ser un comienzo ideal en el camino hacia la dirección de la calidad o TQM, es decir, hacia la mejora continua.

Así, la certificación no debe ser un fin, las empresas deben seguir avanzando en el camino hacia la mejora continua también en otras áreas que influyen realmente sobre la calidad y que no son contempladas por las normas ISO 9000, como son, entre otras, la actividad comercial, gestión administrativa (clientes, proveedores), gestión de recursos humanos, producción (gestión de la producción) o costos. Estas consecuencias, hacen que las normas sean simplemente una guía para iniciar el camino de la calidad.

En la práctica, visualiza la investigadora, todas las empresas se preocupan, en mayor o menor medida, por la calidad. Para muchas, significa casi exclusivamente control de calidad, centrado principalmente en la inspección del producto final, muchas veces simplemente de forma visual, siendo esta práctica más común en las organizaciones que tienen la concepción de que la calidad, en un principio, implica un mayor coste.

En estos casos, las empresas relacionan la aplicación de la serie ISO 9000 con la necesidad de incurrir en mayores costes, en estos casos, las empresas seguirán realizando inspecciones de calidad pero en menor medida. Sin embargo, para otras, la calidad es mucho más. Se tienen desde las que persiguen obtener el certificado según las normas ISO 9000 hasta las que caminan hacia la dirección de la calidad.

\section{Control de los procesos}

Para Münch y García (2005), el control representa una etapa primordial en la gestión, porque aunque una empresa, cuente con excelentes planes, una estructura organizacional adecuada y una dirección eficiente, la gerencia no podrá verificar cuál es su situación real y en consecuencia optimizarse, si no existe un mecanismo de control que verifique si los hechos van acordes con los objetivos planteados.

El controlar un proceso, según Stoner y Wankel (2008), se refiere a como se controlan variables inherentes al mismo para: reducir la variabilidad del producto final, incrementar la eficiencia, reducir impacto ambiental y mantener el proceso dentro de los límites de seguridad que corresponda.

\section{MÉTODO}

La metodología fue de tipo descriptiva, con diseño no experimental, de campo y transeccional. La población quedó constituida por las tres (3) gerencias de yacimientos pertenecientes a las tres (3) empresas mixtas filiales de PDVSA que hacen vida activa en la Costa Oriental del Lago, siendo los sujetos informantes gerentes y líderes, quienes laboraban en los procesos de yacimientos y estaban involucrados a la gestión de calidad en las gerencias reseñadas; para un total de 12 personas.

Para la recolección de datos se empleó la encuesta mediante un cuestionario, 
conformado por 10 ítems con escala de frecuencia. La validez se realizó a través del juicio de expertos, y en su confiabilidad se empleó el método del Coeficiente Alfa de Cronbach, cuyo resultado fue de 0,812 considerado de fuerte confiabilidad. El análisis de los datos se realizó con estadística descriptiva, con base a la media aritmética. En la tabla 1 muestra el baremo de interpretación.

Tabla 1. Baremo para la interpretación de la media aritmética

\begin{tabular}{|c|c|c|}
\hline INTERVALO & CATEGORÍA & DESCRIPCIÓN \\
\hline $4.21-5.00$ & Muy alta aplicación & $\begin{array}{l}\text { Si la aplicación es muy alta o alta se considera una fortaleza para } \\
\text { la gestión de calidad }\end{array}$ \\
\hline $3.41-4.20$ & Alta aplicación & \\
\hline $2.61-3.40$ & Moderada aplicación & $\begin{array}{c}\text { Si la aplicación es moderada se considera una leve fortaleza, la } \\
\text { gestión de calidad requiere mejoras }\end{array}$ \\
\hline $1.81-2.60$ & Baja aplicación & $\begin{array}{l}\text { Si la aplicación es baja o muy baja se considera una debilidad } \\
\text { para la gestión de calidad, por tanto requiere mejoras. }\end{array}$ \\
\hline $1.00-1.80$ & Muy baja aplicación & \\
\hline
\end{tabular}

Fuente: Garrido (2019)

\section{RESULTADOS}

En la tabla 2, se presenta el resumen para la dimensión estrategias de la gestión de calidad, reflejando una media para la dimensión de 2,49, indicando que en las gerencias bajo estudio, se da baja aplicación a las estrategias de la gestión de calidad, siendo esto una debilidad para la gestión que se analiza, lo cual es indicativo de que la misma requiere mejoras, interpretándose de acuerdo al baremo diseñado para tal fin. De manera detallada, se evidencia en estos resultados como todos los indicadores ostentan baja aplicación, a excepción del análisis modo de fallas y efectos categorizado con moderada, lo cual otorga a esta gestión oportunidades de mejora en cada una de las estrategias de la gestión de calidad consideradas en la teoría manejada que sustenta esta dimensión. 
Tabla 2. Dimensión: Estrategias de la gestión de calidad

\begin{tabular}{lcc}
\hline \multicolumn{1}{c}{ INDICADOR } & $\mathrm{X}$ & CATEGORÍA \\
\hline PLANIFICACIÓN DE LA CALIDAD & 2,35 & Baja aplicación/Debilidad \\
DISEÑO DE LA CALIDAD & 2,30 & Baja aplicación/Debilidad \\
DESPLIEGUE FUNCIONAL & 2,35 & Baja aplicación/Debilidad \\
ANÁLISIS MODO DE FALLAS Y EFECTOS & 3,00 & Moderada aplicación/Leve fortaleza \\
CONTROL DE LOS PROCESOS & 2,45 & Baja aplicación/Debilidad \\
DIMENSIÓN & 2,49 & Baja aplicación /Debilidad \\
\hline
\end{tabular}

Fuente: Garrido (2019)

Ahora bien, al ser calificada de baja aplicación la dimensión, no valida lo planteado por Gutiérrez (2010), para quien las estrategias de calidad son una guía para la actividad de la dirección donde se plasman los principios de la calidad para la empresa, detallando entre otras, la intención de satisfacer las necesidades del cliente, las necesidades concretas que han de satisfacerse y la mejora de la calidad. Estas estrategias establecen unos principios a seguir, que indican qué debe hacerse, pero sin explicar cómo debe hacerse, puesto que esto último se describe en un procedimiento.

De igual manera, no validan a la investigadora cuando asume que las estrategias de calidad deben indicar el camino a seguir para que la dirección de la organización pueda encaminar los esfuerzos al logro de los objetivos de calidad, son una guía para la actividad de la dirección en busca de la calidad, con la intención de satisfacer las necesidades del cliente.

Al detalle de los indicadores de esta dimensión, se evidencia, en la tabla 3, moderada aplicación del análisis modo de fallas $y$ efectos $(3,00)$ indicando leves fortalezas al respecto, por ende son oportunidades de mejora. Mientras el resto se ubica en baja aplicación: planificación de la calidad $(2,35)$; diseño de la calidad (2,30); despliegue funcional $(2,35) ; y$ control de los procesos $(2,49)$; siendo estos aspectos debilidades de la gestión de calidad que realizan las gerencias de yacimientos de las empresas mixtas venezolanas, activas en la Costa Oriental del Lago, siendo indicativo de que esta gestión requiere mejoras. 
Tabla 3. Indicadores: Estrategias de la gestión de calidad

\begin{tabular}{cccc}
\hline \multicolumn{4}{c}{ INDICADOR: PLANIFICACIÓN DE LA CALIDAD } \\
\hline $\mathrm{N}^{\circ}$ & ÍTEMS & $\mathrm{X}$ & CATEGORÍA
\end{tabular}

1. Realiza una planificación detallada de cada una de las actividades a ejecutar en la gestión de calidad. 2,40 Baja aplicación/Debilidad

2. El personal participa en la planificación de las actividades a realizar en la gestión de calidad

2,30 Baja aplicación/Debilidad

Promedio Indicador 2,35 Baja aplicación /Debilidad INDICADOR: DISEÑO DE LA CALIDAD

$\mathrm{N}^{\circ} \quad$ ÍTEMS CATEGORÍA

3. Proporcionan la oportunidad al personal para participar en las técnicas que se requieren aplicar en la gestión de calidad.

1,80 Muy baja aplicación/Debilidad

4. Logran certificar los sistemas de aseguramiento externos en calidad

Moderada aplicación/

Leve fortaleza

Promedio Indicador 2,30 INDICADOR: DESPLIEGUE FUNCIONAL

$\mathrm{N}^{\circ} \quad$ ITEMS

5. Consideran dentro de las actividades de gestión de calidad las necesidades de los clientes de la empresa.

6. Establecen fuentes de información para futuros procesos (diseños, servicios, mejoras) proporcionando un sistema fiable del seguimiento del producto o servicio a través del proceso.

\section{CATEGORÍA}

2,50 Baja aplicación/Debilidad

2,20 Baja aplicación/Debilidad

Promedio Indicador 2,35 Baja aplicación/Debilidad INDICADOR: ANÁLISIS MODO DE FALLAS Y EFECTOS

$\mathrm{N}^{\circ} \quad$ ITEMS

7. Realizan un análisis de modo de fallas y efectos para optimizar la gestión de calidad en las actividades que se ejecutan.

8. Evalúan la confiabilidad de sus procesos, revisando modos de falla esperados, para encontrar los efectos de la fallas en la salida de sus procesos
$\mathrm{X}$ CATEGORÍA

3,10

Moderada aplicación/ Leve fortaleza

Moderada aplicación/ Leve fortaleza

Moderada aplicación/ Leve fortaleza

\section{INDICADOR: CONTROL DE LOS PROCESOS}

\section{$\mathrm{N}^{\circ} \quad$ ITEMS}

9. Verifican la eficacia de las actividades planificadas en la 2,20 gestión de calidad.

10. Logran prever los posibles errores que se pueden presentar 2,70 en las actividades que ejecutan
X CATEGORÍA

Baja aplicación/Debilidad

Moderada aplicación/ Leve fortaleza

Promedio Indicador 2,45
Baja aplicación /Debilidad Baja aplicación /Debilidad

Fuente: Garrido (2019) 
Estos resultados ponen en evidencia que, en las gerencias estudiadas, las estrategias de calidad más usuales en las organizaciones, para lograr la efectividad de la misma, poseen baja aplicación en conjunto, y de manera separada solo la estrategia de análisis modo de fallas y efectos muestra moderada aplicación, al tiempo que el resto posee baja aplicación. Esta situación refleja que la gestión de calidad, aquí analizada, muestra oportunidades de mejora en cada uno de los aspectos medidos a través de los ítems utilizados; dichos aspectos serán la base para establecer acciones estratégicas que orienten la gestión de calidad en las gerencias reseñadas.

De manera general, puede decirse que los resultados no logran validar la teoría que sustenta esta investigación, donde autores como Gutiérrez (2010), Arthur (2010), Benavides (2008), y Hendricks y Singhal (2006), coinciden en que las estrategias de calidad pueden ser establecidas en cada organización de acuerdo a los objetivos trazados en cuanto a calidad se refiere, siendo las más usuales: planificación de la calidad, diseño de la calidad, despliegue funcional, análisis modo de fallas y efectos, y el control de los procesos.

\section{CONCLUSIONES}

En lo que concierne al objetivo, dirigido a identificar las estrategias de la gestión de calidad que aplican, se concluye que: en las gerencias bajo estudio se da baja aplicación a las estrategias de la gestión de calidad, siendo esto una debilidad para la gestión que se analizó, lo cual es indicativo de que la misma requiere mejoras. También se evidenció que todos los indicadores ostentaron baja aplicación, a excepción del análisis modo de fallas y efectos categorizado con moderada, lo cual otorgó a esta gestión oportunidades de mejora en cada una de las estrategias de gestión de calidad consideradas.

Al respecto, entre las estrategias requeridas para sustentar la gestión de calidad, desde la óptica de la teoría que sustenta esta investigación se deben aplicar:

$\checkmark$ Realizar una planificación detallada de cada una de las actividades a ejecutar en la gestión de calidad.

$\checkmark$ Asegurar que se logren certificar los sistemas de aseguramiento externos en calidad.

$\checkmark$ Establecer fuentes de información para futuros procesos (diseños, servicios, mejoras) proporcionando un sistema fiable del seguimiento del producto o servicio a través del proceso.

$\checkmark$ Fijar los mecanismos necesarios para que se realicen análisis de modo de fallas y efectos para optimizar la gestión de calidad en las actividades que se ejecutan.

$\checkmark$ Verificar periódicamente la eficacia de las actividades planificadas en la gestión de calidad.

\section{REFERENCIAS}

Alcalde, P. (2008). Competir por medio de la calidad. Editorial McGraw-Hill, México.

Arthur, A. (2010). Principios de calidad empresarial. Editorial Paninfo. Argentina

Benavides, A. (2008). Gerenciando la calidad en las empresas. Editorial McGraw-Hill, Madrid - España

Collado, R. (2006). Calidad y Competitividad empresarial. Editorial Trotta. España

Cuatrecasas, L. (2010). Lean management, la gestión competitiva por excelencia: implantación progresiva en 7 etapas Editorial Profit. Barcelona

Fernández, U. (2008). Los pronósticos en la calidad. Pearson Educación. México, D.F 
Gutiérrez, H. (2010). Calidad total y productividad. Editorial McGraw-Hill. México

Harendes, M. (2009). Principios de la Administración. Editorial CECSA, México, D.F

Hendricks, D. y Singhal, R. (2006). Dirección de Marketing. Duodécima Edición. Pearson Educación, S.A. México

James, P. (2010). Gestión de la calidad total. Un texto introductorio. Editorial Prentice Hall. Madrid. España

Koontz, H. y Weihrich, K. (2009). Administración Una Perspectiva Global, 12a . Edición, McGraw Hill Interamericana. México, D.F

Marín, D. (2009). Fundamentos de la gestión de calidad. Ediciones CIDEG. Venezuela

Miranda, G., Chamorro, N., y Rubio, G. (2007). Los clientes y su satisfacción integral. Editorial McGraw-Hill. España
Münch, G. y García, H. (2005). Gestión de la Calidad Total: un texto introductorio. Editorial McGraw-Hill. Madrid, España

Muñoz, J. (2009). La gestión integrada: calidad, seguridad y medio ambiente. Segunda edición. Editorial SERFOREM, S. L. España

Norma ISO 9000 (2006). Sistemas de Gestión de la Calidad. Fundamentos y vocabulario. Tercera revisión. Abril 2006

Pérez, P. y Munera, F. (2007). Reflexiones para implementar un sistema de gestión de calidad (ISO 9001:2000) en cooperativas y empresas de economía solidaria. Editorial Universidad Cooperativa de Colombia. Colombia

Stoner, H. y Wankel, G. (2008). Potenciando la calidad en las empresas. Editorial Paidós. México, D.F

Van Ham, J. (2009). Cultura competitiva en las empresas. Ediciones Limusa. México 